\title{
A UTILIZAÇÃO DO GRUPO FOCAL COMO METODOLOGIA QUALITATIVA NA PROMOÇÃO DA SAÚDE
}

\section{THE UTILIZATION OF FOCAL GROUP A QUALITY METHODOLOGY ON HEALTH PROMOTION}

Solange Abrocesi lervolino* Maria Cecilia Focesi Pelicioni**

\begin{abstract}
IERVOLINO, SA.; PELICIONI, MCF. A utilização do grupo focal como metodologia qualitativa na promoção da saúde. Rev Esc Enf USP, v. 35, n.2, p.115-21, jun, 2001.

\section{RESUMO}

O presente trabalho procura demonstrar a importância para a promoção em saúde da utilização de uma técnica de pesquisa qualitativa para diagnóstico denominada grupo focal. É uma técnica de diagnóstico rápida e de baixo custo, utilizada para completar in formações, conhecer atitudes, opiniões, percepções e comportamentos relativos à saúde; para desenvolvimento de programas e para avaliar recursos audiovisuais. A utilização desta metodologia têm se mostrado muito adequada para a fase de diagnóstico e outros eventos de Promoção da Saúde, conforme experiências relatadas que foram vivenciadas pelos autores.
\end{abstract}

PALAVRAS-ChAVE: Grupos focais. Promoção da saúde. Planejamento em saúde.

\section{Educação em saúde. ABSTRACT}

The present work aims at demonstrating the importance of application of a quality research technique for health awareness. Such technique, called focus group, can be widely applied because of its low cost and also because it enables a fast diagnosis. Use complement information supplied by the community, know attitudes, points of view, perceptions and behaviors related to health, in order to develop programs and evaluates audiovisual tools. The application of this methodology has proven very effective for diagnoses and events on Health Awareness, according to experiments already carried out by the authors, which will be reported.

KEYWORDS: Focus grups, Health promotion. Health planing. Health education.

\section{INTRODUÇÃO}

O reconhecimento do direito de que todos os segmentos da população devam ter acesso às informações necessárias, para poder participar da tomada de decisão e da avaliação de programas de saúde, tem levado os administradores e profissionais de saúde a buscar novas estratégias de atuação"

Freqüentemente tem-se constatado divergências entre o que os profissionais de saúde acreditam que a população precisa saber e o que alguns grupos consideram realmente importante.

O entendimento dos comportamentos da população ligados à saúde e à doença se faz cada vez mais necessário e é essencial para o desenvolvimento de ações de prevenção, em parte pelos resultados frustrantes que ações de intervenção baseadas somente no enfoque médico e biológico vêm apresentando nos vários ramos da saúde pública, em parte devido aos resultados negativos dos programas de saúde.

Segundo GILBERT ${ }^{3}$, para modificar comportamentos é necessário primeiro que se entenda o contexto no qual ocorrem os significados e a importância a ele atribuído pelos seus agentes. Enquanto pequena parcela do significado do contexto

\footnotetext{
* Enfa. Mestranda da Faculdade de Saúde Pública da Universidade de São Paulo. E-mail: abroiervolino@mandic.com.br

** Prof. Dr. do Depto. de Prática de Saúde Pública da Faculdade de Saúde Pública da Universidade de São Paulo.E-mail: tatim@usp.br
} 
comportamental é de cunho pessoal e particular, grande parte deste é culturalmente moldado e socialmente construido.

Um dos instrumentos científicos utilizados para a resolução deste problema é o emprego de metodologia de pesquisa que permita ao mesmo tempo aproximação da população e compreensão dos símbolos, dos significados e significantes que esta utiliza na apreensão da realidade.

A literatura internacional sobre pesquisa em saúde das duas últimas décadas revela que grande parte das investigações diagnósticas e avaliativas de programas de saúde, têm sido acompanhadas com muita freqüência de estudos qualitativos, embora utilize também modelos quantitativos experimentais e quase- experimentais de pesquisa. Os procedimentos qualitativos têm sido utilizados quando o objetivo do investigador é verificar como as pessoas avaliam uma experiência, idéia ou evento; como definem um problema e quais opiniões, sentimentos e significados encontram-se associados a determinados fenômenos.

Como técnica de pesquisa qualitativa, o grupo focal obtêm dados a partir de reuniões em grupo com pessoas que representam o objeto de estudo. O grupo focal têm sido utilizado internacionalmente para a estruturação de ações diagnósticas e levantamento de problemas; para o planejamento de atividades educativas, como objeto de promoção em saúde e meio ambiente; podendo ser utilizado também para a revisão do processo de ensino-aprendizagem. Relativamente simples e rápido, o grupo focal parece responder a contento à nova tendência da educação em saúde, que tem se deslocado da perspectiva do indivíduo para a do grupo social e da educação calcada em conteúdos e abordagens universais para a educação centrada na perspectiva cultural de seus possiveis beneficiários. ${ }^{6,13}$

Alguns autores ${ }^{1,4,9}$ afirmam que o grupo focal enfatiza a compreensão dos problemas do ponto de vista dos grupos populacionais, assim como o conhecimento das aspirações da comunidade expressos por ela própria, e que sua utilização é "consistente com a filosofia da Educação em Saúde" por se apoiar no princípio da "participação integral" do educando no processo educativo.

Segundo BASCH${ }^{1}$, o uso do grupo focal pode minimizar o número de programas que resultam em baixa efetividade, ou ainda, pode reduzir o número de iniciativas distorcidas que por estarem embasadas na percepção e nos interesses dos dirigentes (e não da população), serão pouco efetivas do ponto de vista de resolutividade de problemas. O uso do grupo focal, pode ainda servir como forma de aproximação, integração e envolvimento com os participantes. Como técnica diagnóstica, permite o entendimento e o redirecionamento dos programas pela incorporação da perspectiva da população alvo. Em sintese, desenvolver uma pesquisa utilizando o grupo focal é desenvolver um processo, que contém procedimentos que visam a compreensão das experiências do grupo participante, do seu próprio ponto de vista.

\section{METODOLOGIA}

\subsection{Caracteristicas Essenciais do Grupo Focal}

O grupo focal pode ser utilizado no entendimento das diferentes percepções e atitudes acerca de um fato, prática, produto ou serviço. O grupo focal, em geral, não é considerado adequado para estudar a freqüência com que determinados comportamentos ou opiniões ocorrem. Pode ser considerado uma espécie de entrevista de grupo, embora não no sentido de ser um processo onde se alternam perguntas do pesquisador e respostas dos participantes.

A essência do grupo focal consiste justamente na interação entre os participantes e o pesquisador, que objetiva colher dados a partir da discussão focada em tópicos específicos e diretivos (por isso é chamado grupo focal).

É composto por 6 a 10 participantes que não são familiares uns aos outros. Estes participantes são selecionados por apresentar certas características em comum que estão associadas ao tópico que está sendo pesquisado. Sua duração típica é de uma hora e meia ${ }^{5,8}$.

A coleta de dados através do grupo focal tem como uma de suas maiores riquezas basear-se na tendência humana de formar opiniões e atitudes na interação com outros indivíduos. Ele contrasta, nesse sentido, com dados colhidos em questionários fechados ou entrevistas individuais, onde o indivíduo é convocado a emitir opiniões sobre assuntos que talvez nunca tenha pensado anteriormente. As pessoas, em geral, precisam ouvir as opiniões dos outros antes de formar as suas próprias, e constantemente mudam de posição (ou fundamentam melhor sua posição inicial) quando expostas à discussão em grupo. É exatamente este processo que o grupo focal tenta captar 5,8 .

Cabe ao moderador do grupo (geralmente o pesquisador) criar um ambiente propicio para que diferentes percepções e pontos de vista venham à tona, sem que haja nenhuma pressão para que seus participantes votem, cheguem a um consenso ou estabeleçam algum plano conclusivo. Este ambiente relaxado e condutor de troca de experiências e perspectivas deve também ser garantido através de alguns outros cuidados:

a) seus participantes não devem idealmente, pertencer ao mesmo círculo de amizade ou trabalho. Isto visa evitar que a livre expressão de idéias no grupo seja prejudicada pelo temor do impacto (real ou imaginário) que essas opiniões vão ter posteriormente ${ }^{8}$; 
b) seus participantes devem ser homogêneos em termos de características que interfiram radicalmente na percepção do assunto em foco, visando garantir o clima confortável para a troca de experiências e impressões de caráter muitas vezes pessoal. E importante enfatizar, no entanto, que a busca de homogeneidade em algumas características pessoais não deve implicar na busca de homogeneidade da percepção do problema. Se assim fosse, o grupo focal perderia a sua riqueza fundamental, que é o contraste de diferentes perspectivas entre pessoas semelhantes ${ }^{2}$. Enfim, a seleção dos participantes deve ser homóloga e não restritiva.

Em estudos que utilizam o grupo focal, as discussões são conduzidas várias vezes com diferentes grupos, visando identificar tendências e padrões na percepção do que se definiu como foco de estudo. A análise sistemática e cuidadosa das discussões vai fornecer pistas e "insights" sobre a forma como é percebido um produto, serviço ou plano de atividades educativas ou de ações para a promoção em saúde"

\subsection{Emprego do Grupo Focal no Estudo de Questões de Saúde e Educação}

Embora o grupo focal seja uma técnica utilizada há muitos anos, sua utilização na área da saúde é relativamente recente. Proposto primeiramente pelos cientistas sociais MERTON, FISK E KENDALL 7 na década de 50, o grupo focal foi ignorado durante muito tempo pelos pesquisadores universitários ${ }^{8}$. O mesmo não aconteceu com os pesquisadores de marketing, que imediatamente o incorporaram como uma de suas mais valiosas técnicas de pesquisa, seja pelo seu baixo custo, seja pela rapidez com que o grupo focal fornece dados válidos e confiáveis.

$\mathrm{Na}$ área da saúde, essa técnica de pesquisa qualitativa foi mais utilizada a partir de meados de 1980, e praticamente inexistem estudos publicados até 1984. Desde 1990, entretanto, foi expressivo o aumento de pesquisas utilizando este método ${ }^{2}$.

A posição que o grupo focal vai ocupar na investigação varia de acordo com o objetivo do pesquisador: alguns trabalhos usam o grupo focal para ajudar na formulação de questionários para pesquisas quantitativas, outros utilizam em combinação com outros métodos qualitativos, como observação participante ou em entrevistas em profundidade; alguns o combinam com "surveys" e outros o utilizam como método único.

\subsection{Montar, Conduzir e Analisar Dados do Grupo Focal}

\subsubsection{Montagem}

O planejamento e montagem do grupo focal é talvez a parte mais trabalhosa de um estudo que utilize esse método de pesquisa. É claro que, como qualquer outro estudo, as possibilidades a serem consideradas vão depender de dois fatores básicos: o tempo e os recursos financeiros. De qualquer maneira, há demandas que precisam ser respondidas e que referemse basicamente ao recrutamento de participantes; à escolha de um moderador e seus assistentes; à organização de recursos técnicos para a gravação das sessões; à seleção de um local adequado para a realização dos grupos e da contratação de profissionais para transcreverem e analisarem os dados.

O recrutamento dos participantes vai ocorrer depois de clara determinação do grupo social que se quer estudar. É importante ter em mente que a pesquisa qualitativa, como é o caso do grupo focal, não trabalha com amostras probabilísticas e nem visa estudar a freqüência com que determinado comportamento ou opinião ocorre. Trata-se sim, como já foi dito anteriormente, de utilizar o grupo focal no entendimento de como se formam e se diferem as percepções, opiniões e atitudes acerca de um fato, produto ou serviço.

Definidas as características das pessoas ou grupo social a ser estudado, seu recrutamento deve procurar abranger sua variabilidade (etária, de gênero, de classe social, se for o caso) e ter em vista as observações feitas em tópico anterior sobre a importância de se evitar alocar em um só grupo, pessoas do mesmo círculo imediato de convivência ou que apresentem características muito contrastantes. Observadas estas regras básicas, o recrutamento de voluntários pode se dar:

- de modo aleatório pelo telefone;

- através de um breve questionário de "screening" para selecionar os participantes adequados;

- poranúncio dejomal;

- através do atendimento em serviços de saúde ou escolas;

- através de indicações sucessivas de pessoas pertencentes à população alvo do estudo;

- através de informantes-chave da comunidade em questão.

Oferecer algum incentivo aos participantes do grupo (almoço em um restaurante, participação em sorteio, pagamento em dinheiro, amostras de produtos ou ainda, pequenos brindes) pode ser fator de peso para o sucesso do recrutamento. Enfim, em qualquer método que se adote, é recomendado convidar cerca de $20 \%$ a mais de pessoas do que realmente será necessário para a condução de cada grupo focal para se prevenir contra ausências inesperadas de participantes ${ }^{8}$.

O moderador tem o papel fundamental de garantir por meio de uma intervenção ao mesmo tempo 
discreta e firme, que o grupo aborde os tópicos de interesse do estudo, de maneira menos diretiva possivel. Ele deve contar com a presença no grupo de dois colaboradores, um para anotar os acontecimentos de maior interesse para a pesquisa (relator) e outro para auxiliar na observação da comunicação não verbal (observador), como forma de compreender os sentimentos dos participantes sobre os tópicos discutidos e, eventualmente, intervir na condução do grupo ${ }^{5}$.

O local onde o grupo focal vai se realizar deve ser idealmente neutro, acessivel, silencioso (fator importante para a obtenção de fitas bem gravadas), não movimentado e composto de uma sala com cadeiras dispostas em círculo ou com uma mesa retangular ou oval com cadeiras. A utilização de salões de igrejas, escritórios, residências e escolas é comum.

Faz parte do planejamento dos recursos financeiros a previsão de verba para a compra um pequeno gravador, pilhas ( 2 jogos para cada grupo), fitas K7 (em média utiliza-se 2 por grupo, com tempo de 60 minutos de gravação, cada uma), fitas para filmagem (quando for o caso) e determinado montante para o pagamento de profissionais que farão as transcrições das fitas, peças chaves para o relatório final da pesquisa. Se a transcrição pode ser tarefa de profissionais não pertencentes ao grupo de investigadores responsáveis, o mesmo obviamente não acontece com a análise dos dados, que deve ser feita pelo pesquisador.

\subsubsection{A condução do grupo}

O sucesso para a boa condução de um grupo focal começa no momento em que o primeiro participante entra na sala de discussão. Além de receber cada participante de maneira cordial, cabe ao moderador, criar um ambiente agradável de espera e evitar ao máximo que o tema do grupo focal seja abordado precocemente em conversas informais, o que eventualmente pode "esfriar" a discussão no momento formal de coleta de dados. E muito comum também, a distribuição de uma folha de auto-preenchimento visando obter informações básicas sobre os participantes (idade, sexo, profissão), para posterior controle da equipe de pesquisa ${ }^{8}$.

Uma vez iniciados os trabalhos, a palavra cabe primeiramente ao moderador, que vai apresentar-se e também aos outros membros (observador e relator) da equipe. Deverá expor os objetivos da pesquisa e do grupo de forma honesta, rápida e genérica. Segundo MORGAN8, a melhor maneira de introduzir o que se espera daquele grupo é francamente admitir que o moderador está lá para aprender. Só que este aprender deve ser colocado em termos de "entendimento incompleto" e não de total ignorância, o que obviamente pode soar falso. O segundo passo é explicar a forma de funcionamento do grupo. Além das regras gerais, deve ser explicitamente enfatizado que não se busca consenso na discussão a ser empreendida e que a divergência de perspectiva e experiências é extremamente bem vinda.

O terceiro passo é solicitar consentimento ao grupo para efetuar a gravação e filmagem (em alguns casos pode-se utilizar esse recurso como mais um apoio para análise dos dados obtidos durante a aplicação do método) e dar garantia do total sigilo do material obtido. E muito importante que os participantes se sintam seguros de que o material ali obtido será utilizado somente para a pesquisa. Faz-se então uma breve rodada de apresentação dos participantes. Não se admite mais a entrada de pessoas a partir desse ponto. Inicia-se, então, a exploração do foco de estudo.

A condução do grupo focal se dá a partir de um roteiro de tópicos, relacionados primeiramente com as questões de investigação que o projeto em pauta visa responder. Como a proposta do método é desenvolver uma discussão focada em um tema especifico, recomendase que este roteiro contenha entre 3 e 5 tópicos no máximo, planejados com antecedência. Estes tópicos não devem ser expressos ao grupo em forma de questões, mas em forma de "dicas", de pequenos estímulos para introduzir o assunto ${ }^{12}$. Não é incomum a utilização de cartazes, figuras, filmes ou estórias como fontes de estimulo ao grupo.

Durante a condução do grupo, cabe ao moderador exercer os mais variados papéis:

- solicitar esclarecimento ou aprofundamento de pontos específicos;

- conduzir o grupo para o próximo tópico quando um ponto já foi suficientemente explorado;

- estimularos tímidos;

- desestimular os tipos dominadores (que não param de falar);

- finalizar o grupo ${ }^{12}$.

\subsubsection{Análise dos dados}

Os dados colhidos com a utilização da metodologia de grupo focal são de natureza qualitativa. Isto implica na necessidade de analisar os dados também de forma qualitativa, ou seja, não há tratamento estatístico envolvido, mas um conjunto de procedimentos que visam organizar os dados de modo que eles revelem, com a máxima objetividade e isenção possivel, como os grupos em questão percebem e se relacionam com o foco do estudo em pauta.

As duas maneiras básicas de se proceder à análise são o sumário etnográfico e a codificação dos dados via análise de conteúdo ${ }^{8}$. A diferença principal entre estes dois procedimentos é que o primeiro vai repousar nas citações textuais dos participantes do grupo, que vão assim ilustrar os achados principais 
da análise, enquanto o segundo enfatiza a descrição numérica de como determinadas categorias explicativas aparecem ou estão ausentes das discussões, e em quais contextos isto ocorre. Cabe comentar que eles não são excludentes entre si, sendo possivel combiná-los em um só relatório de análise 2 .

"Muitas vezes, o processo de análise acontece de modo simultâneo com a coleta de dados. Por adotar um processo indutivo, em que as categorias e as hipóteses explicativas se formam a partir dos dados, é procedimento habitual de pesquisa qualitativa refletir e analisar resultados parciais, visando adequar melhor os procedimentos de coleta de dados aos objetivos da pesquisa" 2.

\subsection{Grupo Focal como Técnica de Avaliação de Material Didático}

O aumento crescente de números de casos de dengue no Brasil levaram à produção em grande escala de material instrucional, pelo Ministério da Saúde sobre o assunto. Tendo em vista a necessidade de avaliar esse material que foi produzido e distribuído por todas as regiões do país, um projeto piloto foi realizado durante o mês de maio de 1997, na região sudeste, em duas áreas consideradas de risco nas cidades de Santos e São Paulo, Brasil.

Tendo sido elaborados cartazes, vídeos, "folders" e livros para os professores e para os alunos como recursos educativos para apoiar a campanha contra o dengue, que se iniciou com o "Dia Nacional Contra o Dengue", foi solicitado a docentes da Faculdade de Saúde Pública -USP, incluindo um dos autores deste trabalho, a avaliação destes materiais, objetivando verificar:

se a relação entre o texto e as ilustrações eram pertinentes;

se a linguagem era compatível, quanto à acessibilidade, clareza e adequação à população alvo;

se os objetivos educativos foram alcançados;

qual a viabilidade prática das mensagens propostas;

que tipo de participação sugeriam;

se eram motivadores;

se estimulavam a reflexão;

se tinham por base a realidade socioeconômica e cultural da população alvo.

Foram então organizados nove grupos focais em cada uma das cidades acima citadas (num total de 18 grupos) com 6 a 15 pessoas em cada grupo, constituídos por escolares, pais de escolares, donas de casa, informantes chaves da população (membros de associação de bairro, membros de conselho de saúde entre outros), profissionais da saúde (que trabalham em programas de combate ao dengue e outros que não trabalham especificamente nesse programa) e soldados do exército.

Os grupos tiveram duração máxima de duas horas, durante as quais foram discutidos minuciosamente todos os itens programados para avaliar cada material instrucional. O grupo focal foi conduzido por um moderador que seguiu um roteiro pré- estabelecido. Esse roteiro foi preparado a partir dos objetivos do estudo, propondo a discussão do tema e mantendo o grupo focalizado pelo tempo julgado necessário pelo moderador. Com a permissão dos participantes, alguns grupos foram filmados e todos gravados em fita $\mathrm{K} 7$, que posteriormente foram transcritas, permitindo a avaliação qualitativa dos dados obtidos no processo grupal.

O material foi avaliado de acordo com as experiências de vida e de trabalho de cada grupo investigado. Foram identificadas palavras, frases e ilustrações consideradas dificeis para o entendimento da população alvo. As práticas recomendadas nem sempre foram julgadas viáveis e agradáveis para a população às quais se destinavam. Várias sugestões de modificações foram enumeradas, incluindo: os desenhos incompreensiveis do mosquito da dengue de alguns cartazes; a orientação de trocar água por areia nos vasos das plantas; assim como a correção dos erros de grafia na impressão do livro do professor e a idéia de direcionar as mensagens para o grupo a ser atingido, respeitando suas características.

\section{5 o Grupo Focal como Técnica de Diagnóstico Situational}

Vargem Grande Paulista cidade situada a $40 \mathrm{Km}$ do centro da cidade de São Paulo, com economia basicamente agropecuária, tem sido, há muitos anos, objeto de pesquisa e intervenção de docentes e alunos da Faculdade de Saúde Pública - USP.

Foram organizados pelos autores 2 grupos focais com a participação em cada um, de 10 professores, do Ensino Fundamental (num total de 20 professores) da Rede Municipal de Ensino com objetivo de investigar os seus conhecimentos e práticas em relação à educação, à saúde e ao meio ambiente visando subsidiar com essas informações a capacitação desses professores para trabalhar com os novos Parâmetros Curriculares Nacionais nas áreas de Educação em Saúde e Meio Ambiente.

Os grupos tiveram duração média de uma hora e meia cada, sendo conduzidos pelo moderador que manteve o grupo focalizado no objeto da pesquisa, seguindo um roteiro pré estabelecido, composto de itens como:

percepção dos professores em relação à saúde das crianças; 
$\Rightarrow$ conhecimentos dos professores em relação às doenças que as crianças tiveram;

$\Rightarrow$ desenvolvimento de atividades de educação em saúde na escola;

$\Rightarrow$ práticas dos professores em relação ao encaminhamento e atendimento das crianças para as unidades de saúde;

$\Rightarrow$ métodos utilizados pelos professores para o controle e acompanhamento de saúde e doença das crianças na escola;

$\Rightarrow$ sugestões para que a escola possa contribuir para tornar os alunos, os professores, e o município mais saudável.

Foram identificados nos depoimentos dos professoras, relatos sobre a falta de higiene das crianças, com a presença de miíase ("berne"), piolhos, sujidades de pés, mãos e cavidade bucal; existência de situações de riscos de acidentes no ambiente escolar, dificuldades de abordagem de assuntos relacionados à sexualidade; existência de problemas oculares tais como o tracoma e deficiência visual, como também ansiedade pelo despreparo para o atendimento às emergências quando da ocorrência de acidentes com as crianças.

Nestes grupos pudemos perceber que os professores sentem grande necessidade de trabalhar os temas saúde, educação e meio ambiente em conjunto com pais, alunos e equipe de saúde; desejavam, portanto, receber treinamentos específicos por não se sentirem preparados para a efetivação da Promoção da Saúde dentro de seu ambiente de trabalho, a escola.

\subsection{Outras Experiências}

WESTPHAL 12 em artigo escrito para o Boletim da Oficina Sanitária Panamericana, de 1996 relata algumas pesquisas utilizando o método de grupo focal que tiveram como objetivo fazer emergir dos grupos pesquisados a expressão de suas percepções, representações, opiniões e outros dados semelhantes sobre questões relativas a programas de ação básica em saúde e educação materno-infantil, desenvolvimento de recursos humanos, organização e gestão de serviços de saúde. Dentre as pesquisas realizadas pela autora destacam-se três, abaixo descritas.

\subsubsection{Primeira pesquisa}

A primeira pesquisa baseou-se o programa de ações básicas em saúde e educação desenvolvida em todo o Brasil pela Pastoral da Criança.

O objetivo dos grupos focais foi identificar as percepções dos agentes de saúde sobre o serviço prestado à população com relação às ações básicas em saúde e à postura educativa adotada para desenvolver o trabalho; identificar dificuldades sentidas, sugestões e propostas para sua resolução.
Foram realizados 2 grupos focais com 7 participantes cada. Entre outros, os resultados demonstraram que o enfoque central do trabalho das agentes de saúde era o controle do peso e o controle da vacinação. As orientações transmitidas após verificação dos mesmos eram seguidas de ordens e ameaças a respeito das conseqüências do nãoseguimento das normas. O processo educativo desenvolvido pelas agentes de saúde de forma autoritária, paternalista e pouco participativa, foi um dos fatores responsáveis pela pequena diferença de morbidade por doenças diarreicas entre as crianças do programa e as de outros bairros não envolvidos no programa.

\subsubsection{Segunda pesquisa}

O segundo estudo teve como um dos objetivos verificar a opinião da população sobre as mudanças na atenção à saúde decorrentes da implantação do processo de municipalização dos serviços de saúde em dois municípios da região metropolitana de São Paulo, Cotia e Vargem Grande Paulista.

A amostra foi constituída de 172 líderes comunitários, entre homens e mulheres, que participaram de 20 grupos focais, um em cada subregião de saúde dos mesmos municípios (12 grupos em Cotia e 8 em Vargem Grande Paulista).

Através da análise do material obtido nos grupos focais, foi possivel perceber que em relação às ações inovadoras integrantes do processo de municipalização dos serviços de saúde, a população notou mudanças e identificou novas unidades de saúde, porém julgou muito deficiente o atendimento no que diz respeito ao relacionamento e competência técnica dos funcionários (como um todo), e agravamento da falta de recursos diagnósticos e de tratamento.

\subsubsection{Terceira pesquisa}

Envolveu funcionários do Departamento de Saúde de Vargem Grande Paulista, na cidade de São Paulo, para avaliação diagnóstica de um programa educativo para promover a integração dos funcionários e da população para o exercício do controle social no serviço de saúde local.

Foram realizadas 31 reuniões de grupos focais, com 103 funcionários divididos por categoria funcional.

Entre outros os resultados mostraram que houve dificuldades na comunicação interna e externa com a clientela bem como em aceitar a participação popular no controle social do serviço de saúde local; ficou clara também, a falta de consciência do papel educativo dos próprios funcionários, principalmente os de nível universitário em relação ao pessoal auxiliar. 


\section{COMENTÁRIOS FINAIS}

O método do grupo focal discutido neste trabalho, conforme demonstrado nos relatos das experiências, pode portanto, ser utilizado em estudos de saúde pública com diferentes propósitos:

$\Rightarrow$ gerar hipóteses sobre um assunto partir da perspectiva dos informantes selecionados;

$\Rightarrow \quad$ avaliar um serviço ou intervenção de material instrucional;

$\Rightarrow \quad$ fornecer um quadro inicial para estudo de um campo até então não explorado cientificamente;

$\Rightarrow$ como pesquisa exploratória ou como diagnóstico preliminar;

$\Rightarrow \quad$ obter a interpretação de um grupo sobre resultados quantitativos obtidos em estudo prévio;

$\Rightarrow$ contribuir para a montagem e teste de questionários e escalas para projetos de pesquisas quantitativas.

É importante porém que se tenha claro que nem sempre o grupo focal é adequado para todas as situações. Além disso, há dificuldades que devem ser levadas em conta, uma vez que se constituem em pontos fundamentais para o sucesso da técnica, por exemplo, o papel do moderador precisa ser desempenhado com muita flexibilidade e, ao mesmo tempo, muita firmeza na condução dos tópicos. Outra dificuldade que a técnica apresenta é a análise dos dados que devem ser vistos de maneira qualitativa sem se basear nos preceitos da metodologia quantitativa, tais como o tamanho e representatividade da amostra. Isto pode interferir na fidedignidade estatístic11.Temas de natureza muito pessoal e complexa, possivelmente apresentarão resultados decepcionantes se abordados em grupo focal. Assim, a adoção de grupos focais em um determinado projeto deve ser resultado de muita reflexão, como aliás, deve acontecer com a utilização de qualquer método.

Finalmente, cabe ressaltar que atualmente a popularidade do grupo focal na saúde pública reflete a salutar disposição de combinar métodos e técnicas de várias disciplinas para a compreensão de fenômenos que, de modo cada vez mais claro, não conseguem ser captados e analisados por meio do uso de uma única técnica, ou de técnicas que abordem exclusivamente métodos quantitativos de análise.

\section{REFERÊNCIAS}

BIBLIOGRÁFICAS

1.BASCH, CE. Focus group interview: na underutilized reserch techinique for improving theory and practice in health education. Health Educ Q, v. 14, n.4, p.411-48, 1987.

2.CARLINI-COTRIM, B. Potencialidades da técnica qualitativa grupo focal em investigação sobre o abuso de substâncias. Rev Saúde Pública, v.30, n.3, p.285-93, 1996.

3.GILBERT, MJ. The antropologist as alcohologist: qualitatitive perspectives and methods in alcohol research. Int $J$ Addict, v.25, n.2A, p.127-48, 1990-91.

4.GLIK, D, GORDON, A. A focus group methods forformativa research in child survival. An ivorianexample. Int $\mathbf{Q}$ Comm Health Educ, v.8, n.4, p.297-315, 1988.

5.KRUEGER, RA. Focus Group: a pratical guide for appliedresearch, Newbury Park: Sage Publications, 1988.

6. MCKINLAY, JB. Health promotion throug healthy publicpolicy: the contribution of complementary research methods. Can J Public Health, v.83, supl.1, p.11-9,1992.

7.MERTON, RK, FISK, M, KENDALL, PL. The focused interview; a manual of problems and procedures. Glencoe: Il Free Press, 1956.

8.MORGAN, DL. Focus group as qualitative research. Sage university paper series in: Qualitative research methods. Newbury Park: Sage Publications, 1988.

9.RAMIREZ, AG, SHEPPERD, J. The use of focus group in health reserch. Scand. J Primary Healthy Care, suppl.1, p.81-90, 1988.

10.SCRIMSHAW, SCM,HURTADO,E. Rapid assessment procedures for nutrition and primary health care. Latin Am Center Publ, v.11, p. 15-9, 1987.

11.WESTPHAL, MF. Grupo focal - uma técnica de pesquisa qualitativa: exemplo de sua utilização em saúde pública. São Paulo, 1997. / mimeografado/.

12. WESTPHAL, MF, BOGUS, CM, FARIA, MM. Grupos focais: experiências precursoras em programas educativos em saúde no Brasil. Bol Oficina Sanit Panam, v.120, n.6,1996.

13.YACH, D. The use and value of qualitative methods in health reserch in developing countries. Social Sci Med, v.35, n.4, p.603-12, 1992.

\section{Artigo recebido em 10/09/99}

Artigo aprovado em 02/08/00 